\title{
Review
}

\section{Aberrant Dopamine Transmission and Cognitive Dysfunction in Animal Models of Parkinson's Disease}

\author{
M.J. Lelos and S.B. Dunnett* \\ Brain Repair Group, School of Biosciences, Cardiff University, Cardiff, Wales, UK
}

\begin{abstract}
Due to the relative success of therapeutic interventions aimed at treating the overt motor symptoms evident in Parkinson's disease (PD), a greater appreciation of the non-motor aspects of the disease has emerged in recent time. Indeed, evidence suggests that impairments in emotional processing, behavioural control and cognitive function may emerge early in the onset of the disease. Decades of experimental research have seen the development of diverse animal models, all of which have aimed to mimic the characteristic features of the disease process including the dopaminergic neural cell loss, the molecular neuropathology and the concomitant behavioural impairments. The following review provides an overview of the use of animal, particularly rodent, models in the quest to obtain a greater understanding of the role of corticostriatal dopamine in cognitive and neuropsychiatric functions. Given the limitations of using the available rodent models of PD, including altered motor and motivational function, it has become necessary to employ a range of techniques to eke out the precise function of this neurotransmitter in corticostriatal function. Combinations of lesion and pharmacological studies have allowed the assessment of dopamine depletion and precise receptor populations in the learning or expression of a range of executive functions, which has gained us considerable insight into the relationship between the neuropathology that occurs in PD and the resulting impairments in cognitive and neuropsychiatric function.
\end{abstract}

Keywords: Parkinson's disease, animal model, cognition, neuropsychiatric symptoms, executive control, 6-hydroxydopamine, rat, operant tests

\section{INTRODUCTION}

Due to the relative success of therapeutic interventions aimed at treating the overt motor symptoms evident in Parkinson's disease (PD), a greater appreciation of the non-motor aspects of the disease has emerged in recent time. It is now recognised that emotional, neuropsychiatric and cognitive impairments may emerge even before the observable motor dysfunctions. Depending on the severity of the disease, mild

${ }^{*}$ Correspondence to: Stephen B. Dunnett, School of Bioscience, Cardiff University, Museum Avenue, Cardiff, CF10 3AX, Wales, UK. Tel.: +44 2920 875188; Fax: +44 2920 876749; E-mail: Dunnettsb@cf.ac.uk. cognitive impairment, dementia and fronto-executive dysfunction have been shown to manifest as deficits in cognitive flexibility, focussed planning, working memory, and reinforcement learning [1-3]. Furthermore, neuropsychiatric dysfunctions, including the emergence of depressive, anxious and psychotic tendencies [4-6], have been identified as an important aspect of the PD syndrome. These forms of aberrant neural processing are thought to be driven, at least in part, by dopaminergic dysmodulation resulting from degeneration of the ventral mesencephalic neurons of the substantia nigra and ventral tegmental area.

The following review will consider how well the various available animal models of PD recapitulate 
aspects of emotional, neuropsychiatric and cognitive dysfunction manifest in PD patients. Although dopamine (DA) plays a well established role in many aspects of neural function, including processes that relate to motivation, reward, activity, sleep, attention, and learning, its specific role in cognition is still not well understood. The ability to manipulate and induce a diminution of this neurotransmitter in the brains of experimental animals allows the relationship between dopaminergic dysmodulation and the cognitive dysfunction evident in PD to be clarified.

\section{MODELLING PD IN ANIMALS}

Given the range of pathological symptoms and neurobiological alterations observed in PD, from the diverse motor and neuropsychiatric symptoms to the extensive molecular and cellular alterations identified at the neuroanatomical level, modelling PD in rodents has been challenging and undergone multiple transformations in an attempt to mimic the disease process as closely as possible. Early pharmacological models altered DA transmission either by blocking the synthesis of DA (e.g. with $\alpha$-methyltyrosine), by inhibiting the storage of DA (e.g. with reserpine) or by blocking the DA receptors (e.g. with haloperidol). While each of these models could successfully induce specific aspects of motor dysfunction, such as akinesia and bradykinesia [7, 8], the influence of such pharmacological manipulations on other neurotransmitter systems, such as the noradrenaline system, meant that these models lacked the necessary DA specificity and, in turn, resulted in ambiguity regarding the locus of the induced impairments.

While the introduction of the catecholamine neurotoxin 6-hydroxydopmine (6-OHDA) in the 1960's provided a more focused method of targeting DA systems in the basal ganglia, it too was found to impact upon the noradrenaline system $[9,10]$. In an attempt to reduce this influence, pre-lesion injection of the noradrenaline uptake inhibitor, des-methyl imipramine, was used to render the toxin more specific to depletion of the DAergic cells [10]. Using this toxin, DA depletion could be induced along the nigrostriatal pathway via injection into the lateral ventricles, substantia nigra, the medial forebrain bundle or the striatum itself. Intra-striatal infusion of the 6-OHDA toxin was found to produce differential results depending on the locus and quantity of toxin injected. Bilateral infusion into the lateral ventricles induced motor dysfunctions, such as akinesia and catalepsy, disrupted feeding regimes, and produced aphagia and adipsia, which were too severe for prolonged maintenance of the animal [11, 12]. Interestingly, reducing the level of DA depletion was less detrimental to the welfare of the animal, but instead was found to result in spontaneous recovery of the motor and ingestive dysfunctions [13]. Even animals that underwent some recovery of function, however, would often continue to display bradykinesic movements and reduced motivation for appetitive rewards, rendering them difficult to study using operant behavioural paradigms. More focal bilateral striatal injections of 6-OHDA reduce the impact of the toxin on the animals' welfare and allow for evaluation of the regional influence of DA transmission, but the nature of the partial lesion means that spontaneous recovery of the DA depletion and the resultant behavioural effects render this model of limited use without considerable care.

An alternative method of modelling DA depletion was introduced in the form of the unilateral lesion model. Here, injection of 6-OHDA along the nigrostriatal pathway resulted in a profound unilateral striatal depletion of DA. The primary advantage of this model is the ability to contrast behavioural dysfunction on the side of the body contralateral to the lesion with the relatively spared ipsilateral side. Although unilateral injection of 6-OHDA has been shown to alter the biochemical status of the contralateral hemisphere to a small extent [14], the severity of the unilateral DA depletion on the ipsilateral side is sufficient to induce a postural bias and a motor asymmetry that creates a marked tendency for turning behaviour when the animal is activated. For example, systemic injection of d-amphethamine can induce ipsilateral turns of up to $10-15$ per minute over the several hours duration of drug action $[15,16]$. A distinct advantage of the unilateral model is the lack of impact on the animals' health status, with feeding and motivational drives remaining intact. The presence of a relatively intact contralateral hemisphere does, however, largely eliminate the possibility of study cognitive or neuropsychiatric impairments induced by aberrant DA transmission since the intact hemisphere is able to function relatively normally despite the unilateral disruption. Furthermore, the ability to isolate neural processes to one or other hemisphere exclusively is difficult, even using lateralised tasks.

Other neurotoxins are available that also selectively target the ventral mesenchephalic neurons, one of the most widely studied of which has been l-methyl4-phenyl-tetrahydropiridine (MPTP). While a single peripheral injection of MPTP in primates can induce 
acute partial lesions that are capable of spontaneous recovery, multiple injections yield bilateral lesions and a profound Parkinsonian syndrome that is relatively stable, but can give rise to extreme welfare issues. As with 6-OHDA, MPTP can be targeted towards a single hemisphere by injection into the ascending carotid artery, resulting in unilateral denervation of DAergic fibres. Although this model has proved useful for studying potential novel therapeutics in primates, its applicability to rodents is rather more limited. In particular, whereas the MAO-B isoform of monoamine oxidase is the primary substrate for conversion of MPTP into the active neuronal toxin MPP+, MAO-A is the predominant isoform used in catecholamine metabolism in rat; as a consequence, MPTP is considerably less toxic in rats than in primates, humans and mice, and is of limited use in this species. For example, explicit comparison of bilateral injections of 6-OHDA and MPTP into the rat SNpc demonstrated a number of differences between the models with respect to histological and mortality measurements, with the 6-OHDA model presenting considerably greater cell loss, more acute loss of body weight and a higher rate of mortality [17]. To achieve a comparable lesion in rats to that induced in other species it is necessary to infuse the already oxidised molecule MPP+ [18], and that requires central injection into the nigra, bypassing the principle advantage of MPTP, viz. the utility for its peripheral administration. In mice, MPTP is highly toxic and results in profound DA depletion, although the mechanism by which this occurs results in a model that differs considerably from the pathological situation and it is known to also affect other catecholaminergic neurons [19]. That is, rather than induce primary degeneration of the DAergic neurons, MPTP injected into mice alters, at least to some extent, the activity of the tyrosine hydroxylase enzyme, and thereby affects the biosynthesis of DA in otherwise surviving neurons.

The systemic administration of chemical agents, such as paraquat and rotenone, has been found to induce Parkinsonian symptoms. Indeed, paraquat and rotenone, which are used as pesticides, may denote real environmental risks of developing PD [20]. Chronic oral ingestion of paraquat has been shown to reduce levels of DA and its metabolites in the striatum of mice and the suggested mechanism of action is oxidative stress within neurons of the substantia nigra [21]. Rotenone, a mitochondrial complex I inhibitor, has been shown to induce $\alpha$-synuclein accumulations in the substantia nigra and to reduce tyrosine hydroxylase positive neurons in this regions by $15.4 \%$ after chronic intragastric administration in mice, although these pathological alterations did not induce significant DA loss in the striatum [22]. To date, these pre-clinical models of PD have undergone limited behavioural characterisation, and the relatively mild depletions in striatal DA and tyrosine hydroxlase induced by such chemical agents render such models less ideal for evaluating the role of DA in cognitive function than toxin models.

Genetically modified mouse models of PD have been developed based upon the identification of familial disease mutations, for example, in the $\alpha$-synuclein gene [23], leucine-rich repeat kinase 2 (LRRK2), Parkin, DJ-1 and PTEN-induced kinase 1 (PINK1) [24]. Transgenic $\alpha$-synuclein mice demonstrate neuronal inclusions and loss of DA, but not overt motor impairments [25]. While a number of transgenic and knock-in mice have recently been generated and characterised, to date the model that appears to demonstrate the cardinal features of PD most accurately is the mutant (R1441 G) BAC transgenic mouse line [26]. The LRRK2 transgenic mouse model exhibits an agedependent akinesea-like movement impairment, which has been shown to be responsive to apomorphine and L-dopa administration, reduced DA release and abnormalities in the mesencephalic DA neurons and along their nigrostriatal axonal projections.

Overexpression of key proteins implicated in the pathogenesis of PD can also be induced via injection of recombinant adeno-associated viral (AAV) and lentiviral (LV) vectors, which have been shown to efficiently transduce nigral DAergic neurons. Expression of human wild-type or mutated $\alpha$-synuclein in midbrain DAergic neurons has been found to induce a progressive pathology in the nigrostriatal projection, including aberrant protein aggregation, neuronal dysfunction and cell loss [27-29].

While the more recently developed viral vector and genetically modified models of PD will undoubtedly generate insights into the molecular pathology of PD and represent promising models for testing therapeutic strategies and for mimicking motor, cognitive and neuropsychiatric aspects of the disease, the infancy of such techniques means that few models have yet undergone comprehensive behavioural evaluation. Thus, the neurotoxic 6-OHDA model remains the most widely characterised and utilised model to date and, as such, the following consideration of cognitive deficits induced after DA manipulation will focus largely around the neurotoxin model, with consideration of pharmacological interventions and genetically modified lines where appropriate. 


\section{NEUROPSYCHIATRIC FUNCTION}

\section{Parkinson's disease}

PD patients present with a host of neuropsychiatric symptoms that may be manifest before overt motor symptoms and include the emergence of depressive and anxious tendencies [4]. Furthermore, apathy, agitation, sleep disturbances, fatigue and psychosis have been identified as preclinical symptoms of the disease process [5,6]. Although PD patients occasionally present with symptoms of psychosis, including visual hallucinations, delusions and paranoid beliefs [30], they prove very difficult to evaluate in rodent models. Thus, initial attempts to identify the influence of aberrant DA transmission in the manifestation of neuropsychiatric symptoms have focussed largely on modelling the features of depression and anxiety.

\section{Animal models}

Not only does assessing the development of neuropsychiatric symptoms in animal models of PD require a suitable bilateral model, confidence in their existence is confounded by the concomitant emergence of motor impairments. In an attempt to characterise the emotional and cognitive changes that occur in a premotor stage model of PD, Tadaiesky et al. [31] used a bilateral lesion model in which 6-OHDA was injected into the ventrolateral neostriatum. They report increased anhedonic-depressive-like symptoms, assessed using the sucrose consumption and forced swimming tests, and increased anxiogenic behaviour, as revealed by the elevated plus maze task. Cognitive function was examined using the Morris water maze task, which revealed impaired working memory function. Although this battery of tests represents a valid and broad assessment of preclinical symptoms, the inherent motor abnormalities need to be considered. For example, while the number of entries into the open arms of an elevated plus maze differed between lesion and non-lesion rats, critically, the percentage of time spent in the open arms did not differ between cohorts. Since the latter is generally accepted as a sign of anxiogenic tendencies, it may be that the number of arm entries represents a measure of locomotor activity, more so than an index of anxiety. Furthermore, even subtle differences in limb function can affect performance in swimming speed and the postural profile during swimming and these fine motor impairments may not be evident in broad activity measures. Thus, performance on the forced swimming and water maze task may represent a more subtle motor deficit in swimming efficiency or limb function that is not sufficiently controlled for in this battery of tests, rather than assessing the intended neuropsychiatric symptoms.

Vuckovic et al. [32] examined emotional, associative and neuropsychiatric symptoms in the MPTP mouse model of PD, and demonstrated impaired social transmission of food preference along with increased extinction of freezing behaviours after auditory fear conditioning. They did not, however, find evidence of altered anxiogenic or depressive-like behaviours after employing a battery of tasks that included sucrose preference and light-dark preference tests. Interestingly, neuropsychiatric impairments were not evident in the MPTP mouse model despite the fact that it displays changes in both DA and serotonin throughout multiple brain regions including the striatum, amygdala and frontal cortex [32].

One model that appears to mimic some aspects of the neuropsychiatric syndrome evident in PD patients is the VMAT2-deficient mouse line. Taylor et al. [34] conducted a comprehensive evaluation of non-motor functions in this model across a range of ages and the results demonstrate a progressive development of many of the dysfunctions evident in PD, including impaired olfactory discrimination, altered sleep latency and depressive behaviour. Despite demonstrating increased anxiety at 4-6 months of age, by $12-15$ months of age, mutant mice spent a similar amount of time in the open arms of an elevated plus-maze as did wild-type mice. Further investigation of the depressive symptoms is also warranted to ensure that the increased immobility evident in the forced swimming test and tail suspension test is not a consequence of bilaterally impaired limb use. While this model does not strictly mimic DA-depletion, the disruption to DA, NA and 5-HT neurotransmitter systems in multiple brain regions including the striatum, hippocampus and cortex, may represent a reasonable model of PD given the age-dependent emergence of non-motor behavioural symptoms, the loss of the nigrostriatal DA, the accumulation of $\alpha$-synuclein and evidence of locomotor deficits [33-35]. This model does not, however, allow the precise influence of DA dysmodulation or diminution on neuropsychiatric dysfunction to be evaluated.

\section{ASSOCIATIVE LEARNING}

\section{Parkinson's disease}

Associative learning impairments have been identified in Parkinson's patients, depending on the stage 
and severity of the disease, with significantly more deficits manifest at the later stages of the disease [36]. PD patients were found to be impaired on an associative learning task that required learning by trial and error, a task sensitive to frontal lobe dysfunction [37]. Specifically, an analysis of stimulus-stimulus learning in PD patients, in which spatial and object memory were compared, revealed a particular deficit in learning of the spatial task [38, 39].

A comparison of simple and choice reaction time tasks in PD patients, which require the formation of stimulus associations, has revealed an interesting pattern of results. Utilising the asymmetry of DA depletion in PD patients, one study found that reaction time performance with the more affected hand was impaired relative to performance with the less affected hand [40]. Interestingly, however, in a simple choice reaction time task, in which an early stimulus presentation provided information regarding the nature of the subsequently required response, allowing for motor preparation, PD patients were unimpaired in utilising this advance information to enhance their performance and this effect was comparable for both hands [40].

\section{Animal models}

In animal models it has been found that intact DA transmission is essential for the initial acquisition of appetitive behaviours, but after extended training the influence of the DA system is diminished. For example, after minimal training of a CS-reward pairing, rats injected with the D1 antagonist SCH23390 were found to be impaired in initiating approach responses to both a conditioned stimulus and an unconditioned reward [41]. After extensive training, however, D1 receptor blockade resulted in robust responding to the conditioned stimulus, while continuing to impair the unconditioned response. Thus, DA modulation of synaptic plasticity early in training is necessary for the learning of the CS-outcome pairing, but not the maintenance or expression of the conditioned response [41].

Post-training depletion of DA has been found to impact upon stimulus-response performance. Using a choice reaction time task, Brown and Robbins [42] pre-trained rats to respond in one of two lateralized response locations before infusing 6-OHDA unilaterally into the striatum. Post-lesion performance revealed a marked impairment in response times when stimuli were presented on the side of the body contralateral to the lesion and a marked bias for directing responses in the nearer of the two possible response locations. The results represented a deficit in directing responses in contralateral space rather than an inability to detect stimuli [42]. Indeed Carli et al. [43] discuss a similar lateralized impairment after intrastriatal infusion of 6-OHDA as not indicating sensory inattention, but rather representing a form of 'intentional neglect'.

Interestingly, when comparing the impact of striatal DA depletion on simple and choice reaction time tasks, as Rafal et al. [40] did with PD patients, Brown and Robbins [44] report an impairment in motor readiness but not response preparation. Motor readiness was evaluated relative to the pre-lesion performance in which a delay-dependant speeding of response was initially evident, but this delay-dependency was abolished post-DA depletion. Lesion rats, however, continued to demonstrate a benefit when the response was pre-cued in the simple reaction time task, in a similar manner to PD patients. Furthermore, improved motor readiness has been observed on a conditioned motor task after systemic injection of d-amphetamine or intrastriatal infusion of DA [45].

In a further evaluation of lateralized reaction time performance, Dowd and Dunnett [46] demonstrated differential effects of infusing 6-OHDA either intrastriatally or in the medial forebrain bundle. In the former, a mild post-lesion impairment in movement time and accuracy was evident, both of which spontaneously recovered over time, while the latter produced a more profound lateralized impairment with no subsequent recovery of function. Furthermore, an analysis of the immediate post-lesion performance revealed an interesting phenomenon. Namely, despite demonstrating marked and robust impairments in motor function after induction of a $>95 \%$ unilateral DA depletion in the MFB cohort, on the first day or two of post-operative testing, lesion rats were capable of performing as well as control rats on the contralateral side. Only after a few days of operant testing did the impairment manifest. Indeed, when rats were re-introduced to the test apparatus after a six week break, rats again demonstrated a transient alleviation of the response impairment. Given that the mesolimbocortical pathway and the dorsal striatal cell populations, are considered to play a role in reward signalling [47], the authors hypothesised that the lesion-induced contralateral impairment was not purely motoric in nature. Instead, it is suggested that the motor function remains relatively intact, which accounts for the transiently unimpaired post-lesion performance, but that disruption to the reward signalling pathways impaired the rats' ability to maintain the association between the stimulus and the outcome [48]. In essence, the behaviour appeared to resem- 
ble the concept of extinction insofar as performance declined due to a removal of the reward and new learning about the stimulus-response association was formed. The suggestion that reward signalling impairments may occur upstream of the motoric difficulties opens a new avenue for interpretation of seemingly simple functional deficits in complex tasks [48].

\section{HABITUAL VERSIONS GOAL-DIRECTED BEHAVIOUR}

\section{Parkinson's disease}

Recent studies in patients have suggested that altered DA transmission impacts upon the ability to execute both goal-directed and habitual behaviours, although the precise profile of impairment is not yet well established. In a recent report from de Wit et al. [49], PD patients undergoing an instrumental response conflict task demonstrated intact use of stimulus-response associative representations, while the ability to utilise the goal-directed associative components appropriately was dependent on the severity of the disease. Thus, interestingly, despite the fact that patients with PD predominately present with loss of DA in the posterior putamen, the region of the basal ganglia associated with habitual behaviours, de Wit and colleagues suggest that $\mathrm{S}-\mathrm{R}$ behaviours remain intact in $\mathrm{PD}$ patients. In a recent review evaluating the relationship between basal ganglia function and the neuropathology manifest in PD, it is suggested that primarily habitual responding, and to a lesser extent goal-directed behaviour, are likely impaired (albeit differentially) in PD patients and that disruption to the circuitry underlying habitual behaviours can explain much of the loss of automaticity and impaired implicit control mechanisms displayed by PD patients [50].

\section{Animal models}

DAergic mechanisms have long been associated with the development of behavioural autonomy. Classic theories of instrumental learning suggest that two dissociable associative components control the acquisition and expression of behaviour, namely action-outcome and stimulus-response associations. In the former, behaviour is flexible and is mediated by an action-reward association that guides behaviour according to a learned representation of the incentive value of the goal or response outcome [51-53], in the latter, response selection is largely habitual and, thus, occurs independent of the incentive value of the response outcome [54]. Furthermore, subregional differentiation of the striatum has highlighted distinct corticostriatal pathways underlying each form of learning. At the cellular level, the dorsolateral striatum has been implicated in the maintenance of habitual S-R responding, while the dorsomedial striatum supports the more flexible goal-directed behaviours [55-59].

There is considerable evidence of a role for DA in S-R instrumental behaviour. Post-training intracerebral injections of the caudate nucleus with either direct D1 and D2 agonists, or the indirect DA agonist d-amphetamine, have been shown to improved S-R learning on an 8-arm radial maze task [60]. Indeed, amphetamine sensitisation has also been shown to enhance S-R learning by disrupting the acquisition of goal-directed behaviour. In this experiment, pre-training sensitisation to amphetamine induced robust habitual responses after minimal operant training, relative to vehicle-injected rats that remained goal-directed. Post-training sensitisation with the DA agonist, however, did not induce the habitual behaviours and instead performance remained sensitive to the incentive value of the outcome [61].

It has been hypothesised that amphetamine sensitisation alters DA signalling sufficiently to effect a rapid and robust transition from goal-directed to habitual behaviour, but that this neurotransmitter signalling may only be necessary early on in learning, after which behaviour is transferred to and maintained by other, likely cortical, neural systems [62,63]. Wickens et al. [64] suggest two mechanisms by which this change from DA-mediated to DA-independent behaviour may occur. Firstly, it is possible the DA initially influences and modulates task-relevant corticostriatal glutamatergic postsynaptic potentials during the acquisition of an associative pairing. Long-term, however, DA facilitation may no longer be required for the expression of the behaviour to occur, since these glutamatergic synapses might at this stage function sufficiently to not necessitate the DA-enhancement of glutamatergic transmission [64, 65]. Alternatively, with extended training the neural locus of the behaviour may shift such that it is eventually represented by non-DA regions, rendering it less sensitive to DA modulation. Thus, as training proceeds, conditioned representations may shift from cortico-striatal mediation to corticocortical control $[62,64]$.

Direct empirical evidence of the subregional locus of habitual behaviour has been more difficult to obtain in the case of DA signalling. Interestingly, however, in a recent study, repeated exposure to the psychostimulant methamphetamine was shown to increase significantly 
the density of mushroom and thin spines on medium spiny neurons in the dorsolateral region of the striatum. In contrast, the density of mushroom spines in the dorsomedial striatum was found to be reduced [66]. Given that repeated exposure to methamphetamine has been suggested to be capable of inducing a rapid transition from goal-directed to habital performance [61], the authors suggest that the alteration in spiny density, may represent a mechanism by which DA modulation induces a shift between these forms of behaviour.

Injection of the neurotoxin 6-OHDA into the neostriatum of the rat after operant training has rendered diverse results. In an initial study, Faure et al. [67] trained rats to associate two stimuli (tone or light) with two instrumental responses (lever press or chain pull) and each conditioned pair was associated with a reward (either sucrose or grain pellets). After extensive training and subsequent bilateral depletion of DA from the dorsolateral striatum, rats were tested for automaticity by employing a satiety-specific devaluation procedure. In control rats, only the lever press response became habitual, while the chain pull remained goal-directed and sensitive to devaluation procedures. In DAdepleted rats, both responses remained goal-directed, suggesting that dorsolateral DA is implicated in habitual behaviours. However, in a subsequent experiment aimed at assessing the ability of DA agonists to restore habit formation in DA-depleted rats, the same bilateral 6-OHDA lesion failed to disrupt habitual responding during the extinction test [68]. Despite employing a similar paradigm, this time using two levers instead of a chain pull, the only evidence of altered operant performance was during the rewarded test, in which rats were allowed access to the devalued reward and, therefore, not utilising an internal representation of the associative stimuli and the incentive value of the reward. Furthermore, the dissociation between the dorsomedial and the dorsolateral striatal subregions in the DA modulation of habit learning has yet to be evidenced behaviourally.

DAergic innervation of the prelimbic cortex and DMS is essential for maintaining sensitivity to the contingency between an action and its outcome, but not for influencing goal-directed behaviours relative to the current incentive value of a reward. Whereas outcome devaluation manipulations assess the relationship between an action and the value of the reward, outcome contingency tasks gauge the animal's sensitivity to the schedule of reinforcement. That is, they determine whether the animal is able to detect changes in the relationship between the action and the reward presentation by manipulating the delivery of reward (for example, rewards may be delivered at a probability of 0.2 per press or they may be delivered pseudorandomly during the test session but be completely non-contingent upon lever press). Naneix et al. [69] demonstrated an effect of pre-training 6-OHDA lesions of the prelimbic cortex in a contingency degradation paradigm but not on an outcome devaluation test, and infralimbic lesions had no effect on either paradigm. Furthermore, the mixed D1/D2 receptor antagonist flupenthixol was also shown to disrupt sensitivity to contingency degradation after injection into the prelimbic cortex. Thus, DA transmission is an important mediator of associative function in the prelimbic cortex when the contingency between an action and its reward is modulated, but does not appear to be implicated in the development or expression of responses reflecting the incentive value of an outcome. Interestingly, Lex and Hauber [70] report no impact of 6-OHDA lesions of the prelimbic cortex on either contingency degradation or outcome devaluation, but instead reported impaired sensitivity to the contingency between the action and the outcome after 6-OHDA lesion of the DMS. Unlike excitotoxic lesions [55], outcome devaluation was unaffected by DA dennervation of the DMS.

Interestingly, DA has been shown to influence goaldirected behaviour after infusion into the ventromedial prefrontal cortex (vmPFC), but not the dorsomedial prefrontal cortex (dmPFC) [71]. Intact rats were trained to lever press for a sucrose solution under a schedule of reinforcement capable of inducing habitual responding. After conditioned taste aversion treatment, rats that had DA infused into the dmPFC did not alter their habitual responses, while rats infused with DA in the vmPFC demonstrated sensitivity to the incentive value of the reward by increasing responses when the reward was non-devalued and decreasing lever presses in response to the devalued reward. Furthermore, DA enhancement of the vmPFC suppressed responding in a progressive ratio paradigm. Hitchcott and colleagues [71] suggest that infusions of DA in the vmPFC act to modulate attention in a manner that biases the processing of task-relevant information, while suppressing attention towards task-irrelevant information.

\section{ATTENTIONAL SELECTION}

\section{Parkinson's disease}

Attentional capabilities, which are often considered as part of the executive function domain, are known to involve the DAergic system. Impaired attention has been identified in PD patients after evaluation of event 
related potentials [72] and in tasks of spatial attentional orientation [73]. Furthermore, in a cohort of PD patients identified as having mild cognitive impairment, approximately $10 \%$ were found to present with impairments attentional and executive function [2].

\section{Animal models}

Impaired DA transmission and DA-receptor regulation in the striatum and frontal cortex have often been associated with changes in attentional function. As it early as the late 1980's it was reported that 6-OHDA lesions of the rat ventral striatum, which depleted DA in the nucleus accumbens and reduced neocortical noradrenaline, impacted upon attentional processing [74]. Although the resultant neurotransmitter depletion induced only a transient decrease in speed and impulsivity on the 5 choice serial reaction time (5CSRT) task, a stable attenuation of the increased responding typically observed in response to arousing events, such as bursts of white noise and infusion of systemic amphetamine, became evident. In the dorsal striatum, DA depletion was not found to impair accuracy on the 5CSRT task, but instead lengthened response latencies, increased omissions and increased perseverative behaviours. Furthermore, reducing the temporal predictability of the visual stimulus presentation resulted in an impairment in accuracy, a deficit not induced by ventral striatal 6-OHDA lesions and, thus, specific to dorsal striatal function [74, 75]. Indeed, using an instrastriatal bilateral 6-OHDA model, Almaric and colleagues demonstrated impaired attentional performance on a reaction time task, which was shown to be significantly improved by co-administration of the $\mathrm{D}_{2} / \mathrm{D}_{3}$ receptor agonist, piribedil, and L-Dopa [76], demonstrating a role for striatal dopamine in attentional function.

Granon et al. [77] demonstrated a dissociation between $\mathrm{D} 1$ and $\mathrm{D} 2$ receptors in the $\mathrm{mPFC}$ of rats in modulating attentional function. Using the 5CSRT task, they report no effect on performance after bilateral infusion of the D2 antagonist sulpiride into the mPFC. By contrast, infusion of the D1 receptor antagonist SCH 23390 impaired accuracy of attentional performance on the task in rats originally performing with the highest baseline levels of accuracy. Interestingly, impaired accuracy on the 5CSRT task was induced after simultaneous injection of the D1 receptor antagonist SCH 23390 and the NMDA receptor antagonist CCP into the dorsomedial striatum [78]. Not only was this effect found to be specific to visual discrimination and not found to impact upon other aspects of behavioural control including decision time and omissions, each dose of receptor antagonist in and of itself was shown to be ineffective in inducing this attentional impairment.

Taking a different tact, Chudasama and Robbins [79] demonstrated that prefrontal cortical stimulation of D1 receptors was sufficient to improve attentional accuracy on an operant task designed to challenge both attentional and working memory functions. Furthermore, a beneficial effect of the receptor stimulation on short-term working memory was evident in response to stimuli presented after a long-delay, while impaired performance was induced in the short-delayed trials.

\section{COGNITIVE SET SHIFTING}

\section{Parkinson's disease}

Set-shifting requires the ability to learn a rule to solve a task and to subsequently adapt to a new rule or strategy as the contingencies in the task are altered. This paradigm assesses many aspects of executive function, including cognitive flexibility, attentional ability and working memory. In patients, set-shifting is often assessed using the Wisconsin Card Sorting task, which requires patients to sort cards into piles according to a particular feature of the stimulus presented on the cards. The stimulus typically consists of a number of features, including the shape, the colour and the number depicted. As the task contingencies change, participants need to shift to utilising a new rule that requires learning to ignore the previously relevant dimension and to attend to a new dimension (extra-dimensional shift). A large literature has identified impairments in this aspect of executive function in $\mathrm{PD}$ patients [e.g. 80, 81]. Interestingly, Monchi and colleagues demonstrated an involvement of both the striatum and frontal cortical regions in this task [82] and have argued that DA dysmodulation in the cortical regions as well as the corticostriatal pathway impair performance of different aspects of the task [83].

\section{Animal models}

Analogous tasks have been developed for use in rodents that incorporate similar stimulus features, but rather than just assessing extra-dimensional shift performance, intra-dimensional shifts and reversal manipulations are also integrated in the tasks. Reversal 
manipulations require a shift towards responding to the particular intra-dimensional feature that was previously unrewarded; intra-dimensional shifts can be introduced during a new trial set and require the animal to continue to attend to the previously correct feature, but to learn a new discrimination based on novel stimuli. Required responses in the rodent versions of the set-shifting task typically consist of digging in bowls or responding on manipulanda in operant boxes [e.g. 84, 85]. Interestingly, although cognitive set-shifting deficits have been identified and evaluated extensively in PD patients, little data exists that demonstrates set-shifting impairments in animal models of DA dysregulation. For example, 6-OHDA lesions of the caudate in the common marmoset have been shown to either have no influence on performance during a setshifting task [86], or a mild influence manifest as more focused attention in lesion marmosets [87].

Using a maze-based set-shifting task, Floresco et al. [88] demonstrated a role for D2 receptors, but not D4 receptors, in the prefrontal cortex of rats on attentional set-shifting. Infusion of D2 receptor antagonist eticlopride in PFC impaired the rats' ability to switch between using a response strategy and attending to a visual discrimination, regardless of the order in which the shifts were required. In contrast, the D4 antagonist L-745, 870 actually enhanced set-shifting performance. Interestingly, using a series of procedures that manipulated rule-learning and reward contingencies, Stefani and Moghaddam [89] demonstrated a correlation between frontal cortical DA release and the time taken to shift between discrimination rules. Thus, although a role for prefrontal cortical DA in set-shifting can certainly be inferred from the literature, there has yet to be an explicit evaluation of the focal effect of cortical or striatal DA depletion and performance on this task in rodent models.

\section{WORKING MEMORY}

\section{Parkinson's disease}

Another goal-directed aspect of learning and response selection that is influenced or mediated by cortical or striatal DA transmission includes that of working memory. PD patients in the late stages of the disease have been shown to be impaired in verbal, non-spatial and spatial tests of working memory [1]. Specifically, Lewis et al. [3] suggest that the ability to manipulate information in working memory is more affected in PD than the maintenance or retrieval of information from working memory.

\section{Animal models}

A common method of measuring working memory function in rodent models is by employing an uncued spatial water maze task. In this paradigm, animals are placed in a water maze tank containing opaque water and required to find a submerged platform in order to escape. Since placement of the platform changes between sessions, but not between trials, demands are placed upon the working memory system from trial to trial. Although completion of this task requires the use of spatial room cues and has been shown to be sensitive to working memory impairments, use of this test with models of PD may be confounded by the motor dysfunctions induced after DA depletion, with secondary effects on the neglect of the extra-maze cues necessary to navigate in space [90].

After bilateral injection of either 6-OHDA or MPTP into the SNpc, Ferro et al. [17] report impairments in working memory and stimulus-response learning revealed by increased time to reach a submerged platform in the spatial and cued versions of the Morris water maze. Similarly, Tadaiesky et al. [31] reported impaired working memory in a bilateral intrastriatal 6-OHDA model after observing deficits on the water maze task. In a second behavioural measure however, Tadaiesky et al. report increased immobility in a forced swimming test in lesion rats. Thus, given that rats receiving bilateral dopamine depletion present with motor impairments, increased difficulty in the ability to swim to the platform in each version of the water maze task cannot be ruled out, suggesting that the observed impairments may relate more to motor than cognitive function. Furthermore, impaired navigation in the water maze task in rats receiving bilateral 6-OHDA lesions of the MFB or the lateral ventricle has been reported, with evidence of relative differences in the preference to utilise proximal, rather than distal, cues in the lesion cohort, which may account for the performance deficit [90]. Thus, DA-induced motor deficits in swimming efficiency and/or impaired utilisation of extra-maze cues likely influence the profile of observed impairments in these tasks.

There is, however, evidence that DA transmission plays a role in working memory function. For example, by pharmacologically manipulating DA modulation, prefrontal cortical injections of the D1 receptor antagonists SCH 23390 and SCH 39166 in rhesus monkeys were found to impair performance on a delayed response task, a classic task of working memory, without affecting motor function [91]. 
DA depletion in the caudate nucleus of the common marmoset has also been shown to induce deficits in working memory. Interestingly, while DA depletion produced a marked impairment in a spatial delayed response task, a standard task of working memory, performance on an attentional set-shifting task was found to be largely preserved [87]. Thus, while the precise role of DA in working memory remains to be more precisely understood, DA transmission in corticofrontal systems does appear to be implicated in this cognitive function.

\section{BEHAVIOURAL INHIBITION}

\section{Parkinson's disease}

As well as being a disorder of movement initiation, PD has been found to induce broad deficits in motor and cognitive inhibition [92-95]. Thus, the ability to withhold a prepotent response is impaired in PD and has been linked to altered regulation of DA systems $[96,97]$. Although the impairments in response inhibition and conflict resolution in PD patients have largely been attributed to aberrant functionality of the subthalamic nucleus and pre-supplementary motor cortex, respectively, altered DA transmission in striatum may also contribute. This aspect of executive function can be assessed in patients using tests of motor and cognitive control, which include the smooth pursuit task, the Stroop task, the conditional stop signal reaction time task and the Go/NoGo task. In each of these tests, aspects of attentional, cognitive and motor inhibitory control can be measured by evaluating the ability to withhold a response. When presented with conflict situations, both response initiation and response inhibition have found to be impaired in patients with PD, suggesting that a generalized deficit in response control (i.e., selection and suppression) is manifest [93]. Interestingly, in advanced PD, significantly diminished D2 receptor densities have been reported [98, 99], which may contribute to both the response inhibition and activation impairments evident in PD patients, although other neurotransmitter systems including 5-HT and noradrenaline may also play a role [for a review, see 100].

\section{Animal models}

Impaired behavioural inhibition can be defined as an inability to withhold or suppress a response or the expression of impulsive characteristics. The stop signal task has been used recently in rodents to evaluate the contribution of DA signalling in the striatum to the suppression of ongoing responses. Eagle et al. [101] examined the influence of D1 and D2 receptor subtypes in both the dorsomedial striatum and the nucleus accumbens core on performance in the stop-signal task. Their results demonstrated contrasting effects of the D1 receptor antagonist SCH 23390 and the D2 receptor antagonist sulpiride in the dorsomedial striatum, but no effect of either in the ventral striatum. SCH 23390 was found to decrease (i.e., speed up) stop-signal reaction time, while sulpiride increased (i.e., slowed down) both stop- and go- signal reaction times and impaired trial completion when administered at the high doses. Thus, the D1 and D2 receptor subtypes appear to play contrasting, and therefore possibly balancing, roles in controlling behavioural inhibition [101]. Interestingly, atomoxetine, a noradrenaline-specific reuptake inhibitor, has been shown in human and animal studies to increase the stop-signal reaction time on this task [100, 102].

\section{SEQUENCE LEARNING}

\section{Parkinson's disease}

Considerable evidence has amassed suggesting that PD patients demonstrate impairments in sequence learning. Although ultimately able to acquire a new motor sequence, PD patients take considerably longer to learn the actions, an impairment that is more manifest in the later stages of the disease [103]. Price and Shin [104] report impaired performance on a non-motor sequence learning task in PD patients at the moderate, but not early, stage of the disease. Importantly, sequence learning deficits were manifest without any evidence of impaired spatial attention in these patients. Instead, performance on the non-spatial sequence learning task was found to correlate with results of three neuropsychological tests, including the Wisconsin Card Sorting task, which assessed cognitive flexibility and executive functioning.

\section{Animal models}

Studies of sequential learning in both primate and rodent models after altered DA transmission have identified a role for DA in this form of motor learning [105-107]. Using a serial reaction time task, rats were extensively trained to respond to 12 sequentially illuminated stimuli [106]. Measures of response speed and accuracy were also obtained for two sequence disruption manipulations: namely, the inference test, 
whereby the sequence was occasionally switched from sequential to pseudo-random, and the violation test, where only one of the 12 stimuli is presented out of order. Interestingly, no robust effect of DA depletion was evident after post-training lesion [108], but rats that had received bilateral 6-OHDA lesions of the neostriatum prior to training demonstrated impairments in learning. The neostriatal DA depletions largely targeted the dorsomedial region, with an approximate $44-66 \%$ reduction in striatal DA levels. The result was decreased accuracy and slower reaction times during the learning phase than control rats. Furthermore, during the violation test, control rats demonstrated an increased reaction time to the random stimulus, in contrast to lesion rats, which demonstrated less of a difference between the random and sequential stimuli. Thus, the automation of responses is less evident in rats with 6-OHDA lesions, evidencing a role for striatal DA in the learning of a sequential stream of stimuli. Furthermore, impaired learning in this task has been shown to be specific to dorsostriatal, and not ventral striatal, DA diminution [109].

\section{CONCLUSION}

A considerable amount of data has been amassed that suggests a role for corticostriatal DA transmission in several aspects of cognitive processing, including executive and attentional function, goal-directed and habitual behaviours, working memory and many other aspects of learning or behavioural control. It is worth noting, however, that other neurotransmitter systems, including the noradrendergic and cholinergic system are also considerably disrupted in PD $[110,111]$ and these neurochemical pathways have been implicated in many of the neural function that are disrupted in the course of the disease, including working memory and attentional function $[112,113]$. Thus, while aberrant DA transmission may certainly play a role in disrupting aspects of higher cognitive function in PD patients, the important influence of other neurotransmitter systems and, indeed, the interactions between them, likely constitute important contributing factors to the non-motor symptoms of the disease.

Ideally, long-term progressive degeneration of corticostriatal DAergic neurons could be modelled in rodents, but the available techniques for inducing DA diminution do not allow for either progressive evaluation, nor do they come without limitations and confounding impairments. Interestingly, despite technological advances, the development of numerous genetically modified and viral vector models has, to date, yielded relatively limited data on the relationship between the pathological process in PD and behavioural function. More detailed behavioural evaluations may yet yield interesting results in these models, and the development of even more sophisticated models, including the marking of neural pathways with optical or molecular reporters may soon provide further insight into basal ganglia function and dysfunction.

The mostly widely used techniques for modelling PD at present, however, remain the lesion method and pharmacological manipulation. Given the inherent limitations of using the available models of PD, including altered motor and motivational function, it has become necessary to employ a range of complementary techniques to eke out the precise function of the dopamine neurotransmitter system in corticostriatal function. Thus, combinations of pharmacological and lesion studies have allowed the assessment of DA depletion and precise receptor populations in the learning or expression of DA-modulated behaviours, which has gained us considerable insight into the relationship between the neurobiological pathology and neurotransmitter dysregulation that occurs in PD, and the resulting impairments in cognitive or neuropsychiatric function.

\section{ACKNOWLEDGMENTS}

The authors' studies in this area have been funded by the MRC, the Parkinson's Disease Society and the European Union Framework 7 NeuroStemCell and Replaces programmes.

\section{REFERENCES}

[1] Owen AM, Iddon JL, Hodges JR, Summers BA \& Robbins TW (1997) Spatial and non-spatial working memory at different stages of Parkinson's disease. Neuropsychologia, 35, 519-532.

[2] Aarsland D, Bronnick K, Williams-Gray C, Weintraub D, Marder K, Kulisevsky J, Burn D, Barone P, Pagonabarraga J, Allcock L, Santangelo G, Foltynie T, Janvin C, Larsen JP, Barker RA \& Emre M (2010) Mild cognitive impairment in Parkinson disease: A multicenter pooled analysis. Neurology, 75, 1062-1069.

[3] Lewis SJ, Slabosz A, Robbins TW, Barker RA \& Owen AM (2005) Dopaminergic basis for deficits in working memory but not attentional set-shifting in Parkinson's disease. Neuropsychologia, 43, 823-832.

[4] Dias FM, Kummer A, Doyle FC, Harsanyi E, Cardoso F, Fontenelle LF \& Teixeira AL (2011) Psychiatric disorders in primary focal dystonia and in Parkinson's disease. Neuropsychiatr Dis Treat, 7, 111-116.

[5] Aarsland D, Bronnick K, Alves G, Tysnes OB, Pedersen KF, Ehrt U \& Larsen JP (2009) The spectrum of neuropsychi- 
atric symptoms in patients with early untreated Parkinson's disease. J Neurol Neurosurg Psychiatry, 80, 928-930.

[6] Riedel O, Klotsche J, Spottke A, Deuschl G, Forstl H, Henn F, Heuser I, Oertel W, Reichmann H, Riederer P, Trenkwalder C, Dodel R \& Wittchen HU (2010) Frequency of dementia, depression, and other neuropsychiatric symptoms in 1,449 outpatients with Parkinson's disease. J Neurol, 257, 1073-1082.

[7] Colpaert FC (1987) Pharmacological characteristics of tremor, rigidity and hypokinesia induced by reserpine in rat. Neuropharmacology, 26, 1431-1440.

[8] De Ryck M, Schallert T \& Teitelbaum P (1980) Morphine versus haloperidol catalepsy in the rat: A behavioral analysis of postural support mechanisms. Brain Res, 201, 143-172.

[9] Uretsky NJ \& Iversen LL (1969) Effects of 6-hydroxydopamine on noradrenaline-containing neurones in the rat brain. Nature, 221, 557-559.

[10] Breese GR \& Traylor TD (1971) Depletion of brain noradrenaline and dopamine by 6-hydroxydopamine. $\mathrm{Br} \mathrm{J}$ Pharmacol, 42, 88-99.

[11] Ungerstedt U (1971) Adipsia and aphagia after 6-hydroxydopamine induced degeneration of the nigro-striatal dopamine system. Acta Physiol Scand Suppl, 367, 95-122.

[12] Zigmond MJ \& Stricker EM (1972) Deficits in feeding behavior after intraventricular injection of 6-hydroxydopamine in rats. Science, 177, 1211-1214.

[13] Zigmond MJ \& Stricker EM (1973) Recovery of feeding and drinking by rats after intraventricular 6-hydroxydopamine or lateral hypothalamic lesions. Science, 182, 717-720.

[14] Pierucci M, Di Matteo V, Benigno A, Crescimanno G, Esposito E \& Di Giovanni G (2009) The unilateral nigral lesion induces dramatic bilateral modification on rat brain monoamine neurochemistry. Ann N Y Acad Sci, 1155, 316323.

[15] Ungerstedt U \& Arbuthnott GW (1970) Quantitative recording of rotational behavior in rats after 6-hydroxy-dopamine lesions of the nigrostriatal dopamine system. Brain Res, 24, 485-493.

[16] Ungerstedt U (1971) Striatal dopamine release after amphetamine or nerve degeneration revealed by rotational behaviour. Acta Physiol Scand Suppl, 367, 49-68.

[17] Ferro MM, Bellissimo MI, Anselmo-Franci JA, Angellucci ME, Canteras NS \& Da Cunha C (2005) Comparison of bilaterally 6-OHDA- and MPTP-lesioned rats as models of the early phase of Parkinson's disease: Histological, neurochemical, motor and memory alterations. $J$ Neurosci Methods, 148, 78-87.

[18] Sirinathsinghji DJ, Heavens RP, Richards SJ, Beresford IJ \& Hall MD (1988) Experimental hemiparkinsonism in the rat following chronic unilateral infusion of MPP+ into the nigrostriatal dopamine pathway-I. Behavioural, neurochemical and histological characterization of the lesion. Neuroscience, 27, 117-128.

[19] Kitt CA, Cork LC, Eidelberg E, Joh TH \& Price DL (1987) Injury of catecholaminergic neurons after acute exposure to MPTP. Annals of the New York Academy of Sciences, 495, 730-731.

[20] Tanner CM, Kamel F, Ross GW, Hoppin JA, Goldman SM, Korell M, Marras C, Bhudhikanok GS, Kasten M, Chade AR, Comyns K, Richards MB, Meng C, Priestley B, Fernandez HH, Cambi F, Umbach DM, Blair A, Sandler DP \& Langston JW (2011) Rotenone, paraquat, and Parkinson's disease. Environ Health Perspect, 119, 866-872.

[21] Ren JP, Zhao YW \& Sun XJ (2009) Toxic influence of chronic oral administration of paraquat on nigrostriatal dopaminergic neurons in C57BL/6 mice. Chin Med J(Engl), 122, 2366-2371.

[22] Pan-Montojo F, Anichtchik O, Dening Y, Knels L, Pursche S, Jung R, Jackson S, Gille G, Spillantini MG, Reichmann H \& Funk RH (2010) Progression of Parkinson's disease pathology is reproduced by intragastric administration of rotenone in mice. Plos One, $\mathbf{5}$, e8762.

[23] Polymeropoulos MH, Lavedan C, Leroy E, Ide SE, Dehejia A, Dutra A, Pike B, Root H, Rubenstein J, Boyer R, Stenroos ES, Chandrasekharappa S, Athanassiadou A, Papapetropoulos T, Johnson WG, Lazzarini AM, Duvoisin RC, Di Iorio G, Golbe LI \& Nussbaum RL (1997) Mutation in the alpha-synuclein gene identified in families with Parkinson's disease. Science, 276, 2045-2047.

[24] Henchcliffe C \& Beal MF (2008) Mitochondrial biology and oxidative stress in Parkinson disease pathogenesis. Nat Clin Pract Neurol, 4, 600-609.

[25] Masliah E, Rockenstein E, Veinbergs I, Mallory M, Hashimoto M, Takeda A, Sagara Y, Sisk A \& Mucke L (2000) Dopaminergic loss and inclusion body formation in alpha-synuclein mice: Implications for neurodegenerative disorders. Science, 287, 1265-1269.

[26] Li Y, Liu W, Oo TF, Wang L, Tang Y, Jackson-Lewis V, Zhou C, Geghman K, Bogdanov M, Przedborski S, Beal MF, Burke RE \& Li C (2009) Mutant LRRK2(R1441G) BAC transgenic mice recapitulate cardinal features of Parkinson's disease. Nat Neurosci, 12, 826-828.

[27] Kirik D \& Bjorklund A (2003) Modeling CNS neurodegeneration by overexpression of disease-causing proteins using viral vectors. Trends Neurosci, 26, 386-392.

[28] Kirik D, Rosenblad C, Burger C, Lundberg C, Johansen TE, Muzyczka N, Mandel RJ \& Bjorklund A (2002) Parkinsonlike neurodegeneration induced by targeted overexpression of alpha-synuclein in the nigrostriatal system. J Neurosci, 22, 2780-2791.

[29] Maingay M, Romero-Ramos M \& Kirik D (2005) Viral vector mediated overexpression of human alpha-synuclein in the nigrostriatal dopaminergic neurons: A new model for Parkinson's disease. CNS Spectr, 10, 235-244.

[30] Williams-Gray CH, Foltynie T, Lewis SJ \& Barker RA (2006) Cognitive deficits and psychosis in Parkinson's disease: A review of pathophysiology and therapeutic options. CNS Drugs, 20, 477-505.

[31] Tadaiesky MT, Dombrowski PA, Figueiredo CP, CargninFerreira E, Da Cunha C \& Takahashi RN (2008) Emotional, cognitive and neurochemical alterations in a premotor stage model of Parkinson's disease. Neuroscience, 156, 830-840.

[32] Vuckovic MG, Wood RI, Holschneider DP, Abernathy A, Togasaki DM, Smith A, Petzinger GM \& Jakowec MW (2008) Memory, mood, dopamine, and serotonin in the 1-methyl-4-phenyl-1,2,3,6-tetrahydropyridine-lesioned mouse model of basal ganglia injury. Neurobiol Dis, 32, 319-327.

[33] Taylor TN, Caudle WM \& Miller GW (2011) VMAT2deficient mice display nigral and extranigral pathology and motor and nonmotor symptoms of Parkinson's disease. Parkinsons Dis, 2011, 124165.

[34] Taylor TN, Caudle WM, Shepherd KR, Noorian A, Jackson CR, Iuvone PM, Weinshenker D, Greene JG \& Miller GW (2009) Nonmotor symptoms of Parkinson's disease revealed in an animal model with reduced monoamine storage capacity. J Neurosci, 29, 8103-8113.

[35] Vernon AC (2009) Mice with reduced vesicular monoamine storage content display nonmotor features of Parkinson's disease. J Neurosci, 29, 12842-12844. 
[36] Canavan AG, Passingham RE, Marsden CD, Quinn N, Wyke M \& Polkey CE (1989) The performance on learning tasks of patients in the early stages of Parkinson's disease. Neuropsychologia, 27, 141-156.

[37] Vriezen ER \& Moscovitch M (1990) Memory for temporal order and conditional associative-learning in patients with Parkinson's disease. Neuropsychologia, 28, 1283-1293.

[38] Postle BR, Jonides J, Smith EE, Corkin S \& Growdon JH (1997) Spatial, but not object, delayed response is impaired in early Parkinson's disease. Neuropsychology, 11, 171-179.

[39] Postle BR, Locascio JJ, Corkin S \& Growdon JH (1997) The time course of spatial and object learning in Parkinson's disease. Neuropsychologia, 35, 1413-1422.

[40] Rafal RD, Friedman JH \& Lannon MC (1989) Preparation of manual movements in hemiparkinsonism. J Neurol Neurosurg Psychiatry, 52, 399-402.

[41] Choi WY, Balsam PD \& Horvitz JC (2005) Extended habit training reduces dopamine mediation of appetitive response expression. J Neurosci, 25, 6729-6733.

[42] Brown VJ \& Robbins TW (1989) Deficits in response space following unilateral striatal dopamine depletion in the rat. $J$ Neurosci, 9, 983-989.

[43] Carli M, Jones GH \& Robbins TW (1989) Effects of unilateral dorsal and ventral striatal dopamine depletion on visual neglect in the rat: A neural and behavioural analysis. Neuroscience, 29, 309-327.

[44] Brown VJ \& Robbins TW (1991) Simple and choice reaction time performance following unilateral striatal dopamine depletion in the rat, Impaired motor readiness but preserved response preparation. Brain, 114(Pt 1B), 513-525.

[45] Baunez C, Nieoullon A \& Amalric M (1995) Dopamine and complex sensorimotor integration: Further studies in a conditioned motor task in the rat. Neuroscience, 65, 375-384.

[46] Dowd E \& Dunnett SB (2005) Comparison of 6-hydroxydopamine-induced medial forebrain bundle and nigrostriatal terminal lesions in a lateralised nose-poking task in rats. Behav Brain Res, 159, 153-161.

[47] Schultz W (2001) Reward signaling by dopamine neurons. Neuroscientist, 7, 293-302.

[48] Dowd E \& Dunnett SB (2007) Movement without dopamine: Striatal dopamine is required to maintain but not to perform learned actions. Biochem Soc Trans, 35, 428-432.

[49] de Wit S, Barker RA, Dickinson AD \& Cools R (2011) Habitual versus goal-directed action control in Parkinson disease. J Cogn Neurosci, 23, 1218-1229.

[50] Redgrave P, Rodriguez M, Smith Y, Rodriguez-Oroz MC, Lehericy S, Bergman H, Agid Y, DeLong MR \& Obeso JA (2010) Goal-directed and habitual control in the basal ganglia: Implications for Parkinson's disease. Nat Rev Neurosci, 11, 760-772.

[51] Dickinson A (1981) Conditioning and associative learning. Br Med Bull, 37, 165-168.

[52] Colwill RM \& Rescorla RA (1990) Effect of reinforcer devaluation on discriminative control of instrumental behavior. J Exp Psychol Anim Behav Process, 16, 40-47.

[53] Balleine B, Ball J \& Dickinson A (1994) Benzodiazepineinduced outcome revaluation and the motivational control of instrumental action in rats. Behav Neurosci, 108, 573-589.

[54] Dickinson A, Balleine B, Watt A, Gonzalez F \& Boakes RA (1995) Motivational control after extended instrumental training. Animal Learning \& Behavior, 23, 197-206.

[55] Yin HH, Ostlund SB, Knowlton BJ \& Balleine BW (2005) The role of the dorsomedial striatum in instrumental conditioning. European Journal of Neuroscience, 22, 513523.
[56] Yin HH \& Knowlton BJ (2006) The role of the basal ganglia in habit formation. Nat Rev Neurosci, 7, 464-476.

[57] Yin HH, Knowlton BJ \& Balleine BW (2004) Lesions of dorsolateral striatum preserve outcome expectancy but disrupt habit formation in instrumental learning. Eur J Neurosci, 19, 181-189.

[58] Yin HH, Knowlton BJ \& Balleine BW (2005) Blockade of NMDA receptors in the dorsomedial striatum prevents action-outcome learning in instrumental conditioning. Eur J Neurosci, 22, 505-512.

[59] Yin HH, Knowlton BJ \& Balleine BW (2006) Inactivation of dorsolateral striatum enhances sensitivity to changes in the action-outcome contingency in instrumental conditioning. Behav Brain Res, 166, 189-196.

[60] Packard MG \& White NM (1991) Dissociation of hippocampus and caudate nucleus memory systems by posttraining intracerebral injection of dopamine agonists. Behav Neurosci, 105, 295-306.

[61] Nelson A \& Killcross S (2006) Amphetamine exposure enhances habit formation. J Neurosci, 26, 3805-3812.

[62] Ashby FG, Turner BO \& Horvitz JC (2010) Cortical and basal ganglia contributions to habit learning and automaticity. Trends Cogn Sci, 14, 208-215.

[63] Costa RM (2007) Plastic corticostriatal circuits for action learning: what's dopamine got to do with it? Ann N Y Acad Sci, 1104, 172-191.

[64] Wickens JR, Horvitz JC, Costa RM \& Killcross S (2007) Dopaminergic mechanisms in actions and habits. JNeurosci, 27, 8181-8183

[65] Horvitz JC (2002) Dopamine gating of glutamatergic sensorimotor and incentive motivational input signals to the striatum. Behav Brain Res, 137, 65-74.

[66] Jedynak JP, Uslaner JM, Esteban JA \& Robinson TE (2007) Methamphetamine-induced structural plasticity in the dorsal striatum. Eur J Neurosci, 25, 847-853.

[67] Faure A, Haberland U, Conde F \& El Massioui N (2005) Lesion to the nigrostriatal dopamine system disrupts stimulus-response habit formation. J Neurosci, 25, 2771-2780.

[68] Faure A, Leblanc-Veyrac P \& El Massioui N (2010) Dopamine agonists increase perseverative instrumental responses but do not restore habit formation in a rat model of Parkinsonism. Neuroscience, 168, 477-486.

[69] Naneix F, Marchand AR, Di Scala G, Pape JR \& Coutureau E (2009) A role for medial prefrontal dopaminergic innervation in instrumental conditioning. J Neurosci, 29, 6599-6606.

[70] Lex B \& Hauber W (2010) The role of dopamine in the prelimbic cortex and the dorsomedial striatum in instrumental conditioning. Cereb Cortex, 20, 873-883.

[71] Hitchcott PK, Quinn JJ \& Taylor JR (2007) Bidirectional modulation of goal-directed actions by prefrontal cortical dopamine. Cereb Cortex, 17, 2820-2827.

[72] Solis-Vivanco R, Ricardo-Garcell J, Rodriguez-Camacho M, Prado-Alcala RA, Rodriguez U, Rodriguez-Violante M \& Rodriguez-Agudelo Y (2011) Involuntary attention impairment in early Parkinson's disease: An event-related potential study. Neurosci Lett, 495, 144-149.

[73] Nys GM, Santens P \& Vingerhoets G (2010) Horizontal and vertical attentional orienting in Parkinson's disease. Brain Cogn, 74, 179-185.

[74] Cole BJ \& Robbins TW (1989) Effects of 6-hydroxydopamine lesions of the nucleus accumbens septi on performance of a 5-choice serial reaction time task in rats: implications for theories of selective attention and arousal. Behav Brain Res, 33, 165-179. 
[75] Baunez C \& Robbins TW (1999) Effects of dopamine depletion of the dorsal striatum and further interaction with subthalamic nucleus lesions in an attentional task in the rat. Neuroscience, 92, 1343-1356.

[76] Turle-Lorenzo N, Maurin B, Puma C, Chezaubernard C, Morain P, Baunez C, Nieoullon A \& Amalric M (2006) The dopamine agonist piribedil with L-DOPA improves attentional dysfunction: Relevance for Parkinson's disease. $J$ Pharmacol Exp Ther, 319, 914-923.

[77] Granon S, Passetti F, Thomas KL, Dalley JW, Everitt BJ \& Robbins TW (2000) Enhanced and impaired attentional performance after infusion of D1 dopaminergic receptor agents into rat prefrontal cortex. J Neurosci, 20, 1208-1215.

[78] Agnoli L \& Carli M (2011) Synergistic interaction of dopamine $\mathrm{D}(1)$ and glutamate N-methyl-d-aspartate receptors in the rat dorsal striatum controls attention. $\mathrm{Neu}$ roscience, 185, 39-49.

[79] Chudasama Y \& Robbins TW (2004) Dopaminergic modulation of visual attention and working memory in the rodent prefrontal cortex. Neuropsychopharmacology, 29, 1628-1636.

[80] Gotham AM, Brown RG \& Marsden CD (1988) 'Frontal' cognitive function in patients with Parkinson's disease 'on' and 'off' levodopa. Brain, 111(Pt 2), 299-321.

[81] Beatty WW, Staton RD, Weir WS, Monson N \& Whitaker HA (1989) Cognitive disturbances in Parkinson's disease. $J$ Geriatr Psychiatry Neurol, 2, 22-33.

[82] Monchi O, Petrides M, Petre V, Worsley K \& Dagher A (2001) Wisconsin Card Sorting revisited: Distinct neural circuits participating in different stages of the task identified by event-related functional magnetic resonance imaging. $J$ Neurosci, 21, 7733-7741.

[83] Monchi O, Petrides M, Doyon J, Postuma RB, Worsley K \& Dagher A (2004) Neural bases of set-shifting deficits in Parkinson's disease. J Neurosci, 24, 702-710.

[84] Birrell JM \& Brown VJ (2000) Medial frontal cortex mediates perceptual attentional set shifting in the rat. J Neurosci, 20, 4320-4324.

[85] Oswald CJ, Yee BK, Rawlins JN, Bannerman DB, Good M \& Honey RC (2001) Involvement of the entorhinal cortex in a process of attentional modulation: Evidence from a novel variant of an IDS/EDS procedure. Behav Neurosci, 115, 841849.

[86] Crofts HS, Dalley JW, Collins P, Van Denderen JC, Everitt BJ, Robbins TW \& Roberts AC (2001) Differential effects of 6-OHDA lesions of the frontal cortex and caudate nucleus on the ability to acquire an attentional set. Cereb Cortex, $\mathbf{1 1}$, 1015-1026.

[87] Collins P, Wilkinson LS, Everitt BJ, Robbins TW \& Roberts AC (2000) The effect of dopamine depletion from the caudate nucleus of the common marmoset (Callithrix jacchus) on tests of prefrontal cognitive function. Behav Neurosci, 114, 3-17.

[88] Floresco SB, Magyar O, Ghods-Sharifi S, Vexelman C \& Tse MT (2006) Multiple dopamine receptor subtypes in the medial prefrontal cortex of the rat regulate set-shifting. Neuropsychopharmacology, 31, 297-309.

[89] Stefani MR \& Moghaddam B (2006) Rule learning and reward contingency are associated with dissociable patterns of dopamine activation in the rat prefrontal cortex, nucleus accumbens, and dorsal striatum. J Neurosci, 26, 8810-8818.

[90] Whishaw IQ \& Dunnett SB (1985) Dopamine depletion, stimulation or blockade in the rat disrupts spatial navigation and locomotion dependent upon beacon or distal cues. Behav Brain Res, 18, 11-29.
[91] Sawaguchi T \& Goldman-Rakic PS (1991) D1 dopamine receptors in prefrontal cortex: Involvement in working memory. Science, 251, 947-950.

[92] Beste C, Dziobek I, Hielscher H, Willemssen R \& Falkenstein M (2009) Effects of stimulus-response compatibility on inhibitory processes in Parkinson's disease. Eur J Neurosci, 29, 855-860.

[93] Obeso I, Wilkinson L, Casabona E, Bringas ML, Alvarez M, Alvarez L, Pavon N, Rodriguez-Oroz MC, Macias R, Obeso JA \& Jahanshahi M (2011) Deficits in inhibitory control and conflict resolution on cognitive and motor tasks in Parkinson's disease. Exp Brain Res, 212, 371-383.

[94] Henderson T, Georgiou-Karistianis N, White O, Millist L, Williams DR, Churchyard A \& Fielding J (2011) Inhibitory control during smooth pursuit in Parkinson's disease and Huntington's disease. Mov Disord. doi: 10.1002/mds.23757 [Epub ahead of print].

[95] Colzato LS, van den Wildenberg WP, van Wouwe NC, Pannebakker MM \& Hommel B (2009) Dopamine and inhibitory action control: Evidence from spontaneous eye blink rates. Exp Brain Res, 196, 467-474.

[96] Aron AR (2007) The neural basis of inhibition in cognitive control. Neuroscientist, 13, 214-228.

[97] Cools R (2008) Role of dopamine in the motivational and cognitive control of behavior. Neuroscientist, 14, 381-395.

[98] Hwang WJ, Yao WJ, Wey SP, Shen LH \& Ting G (2002) Downregulation of striatal dopamine D2 receptors in advanced Parkinson's disease contributes to the development of motor fluctuation. Eur Neurol, 47, 113-117.

[99] Antonini A, Schwarz J, Oertel WH, Pogarell O \& Leenders KL (1997) Long-term changes of striatal dopamine D2 receptors in patients with Parkinson's disease: A study with positron emission tomography and [11C]raclopride. Mov Disord, 12, 33-38.

[100] Eagle DM, Bari A \& Robbins TW (2008) The neuropsychopharmacology of action inhibition: Cross-species translation of the stop-signal and go/no-go tasks. Psychopharmacology (Berl), 199, 439-456.

[101] Eagle DM, Wong JC, Allan ME, Mar AC, Theobald DE \& Robbins TW (2011) Contrasting roles for dopamine D1 and D2 receptor subtypes in the dorsomedial striatum but not the nucleus accumbens core during behavioral inhibition in the stop-signal task in rats. J Neurosci, 31, 73497356.

[102] Robinson ES, Eagle DM, Mar AC, Bari A, Banerjee G, Jiang X, Dalley JW \& Robbins TW (2008) Similar effects of the selective noradrenaline reuptake inhibitor atomoxetine on three distinct forms of impulsivity in the rat. Neuropsychopharmacology, 33, 1028-1037.

[103] Stephan MA, Meier B, Zaugg SW, Kaelin-Lang A (2011) Motor sequence learning performance in Parkinson's disease patients depends on the stage of disease. Brain Cogn, 75, 135-140.

[104] Price A \& Shin JC (2009) The impact of Parkinson's disease on sequence learning: Perceptual pattern learning and executive function. Brain Cogn, 69, 252-261.

[105] Levesque M, Bedard MA, Courtemanche R, Tremblay PL, Scherzer P \& Blanchet PJ (2007) Raclopride-induced motor consolidation impairment in primates: Role of the dopamine type- 2 receptor in movement chunking into integrated sequences. Exp Brain Res, 182, 499-508.

[106] Eckart MT, Huelse-Matia MC, McDonald RS \& Schwarting RK (2010) 6-hydroxydopamine lesions in the rat neostriatum impair sequential learning in a serial reaction time task. Neurotox Res, 17, 287-298. 
[107] Tremblay PL, Bedard MA, Levesque M, Chebli M, Parent M, Courtemanche R \& Blanchet PJ (2009) Motor sequence learning in primate: Role of the D2 receptor in movement chunking during consolidation. Behav Brain Res, 198, 231239.

[108] Domenger D \& Schwarting RK (2008) Effects of neostriatal 6-OHDA lesion on performance in a rat sequential reaction time task. Neurosci Lett, 444, 212-216.

[109] Eckart MT, Huelse-Matia MC, Loer D \& Schwarting RK (2010) Acquisition and performance in a rat sequential reaction time task is not affected by subtotal ventral striatal 6-OHDA lesions. Neurosci Lett, 476, 27-31.

[110] Zweig RM, Jankel WR, Hedreen JC, Mayeux R \& Price DL (1989) The pedunculopontine nucleus in Parkinson's disease. Ann Neurol, 26, 41-46.
[111] Ferrer I (2011) Neuropathology and neurochemistry of nonmotor symptoms in Parkinson's disease. Parkinsons Dis, 2011, 708404.

[112] Hasselmo ME \& Sarter M (2011) Modes and models of forebrain cholinergic neuromodulation of cognition. Neuropsychopharmacology, 36, 52-73.

[113] Kehagia AA, Murray GK \& Robbins TW (2010) Learning and cognitive flexibility: Frontostriatal function and monoaminergic modulation. Curr Opin Neurobiol, 20, 199204. 